\title{
How Mindreading Computer Work And How It Is Useful In Different Working Areas?
}

\author{
KomalTomar \\ DIT University \\ Dehradun,India
}

\begin{abstract}
Mind reading is the ability to infer other people's mental state and use that to make sense of and predict their behavior and also to understand both oneself and other agents in terms of beliefs, desires and intentions[15]. A lack of or impairment in the theory of mind (mindblindness) is thought to be the primary inhibitor of emotion understanding and social intelligence in individuals with autism[6]. The goal in building mind reading machines is to enable computer technologies to understand and react to people's emotions and mental states. In this paper we present how to read minds using different techniques. The paper presents a mind-reading architecture based on an Eye Language Interpreter and Baron-Cohen's Mindreading System. This architecture enables the recognition and prediction of complex mental states, allowing for more natural man-machine interaction. This paper also presents where mind reading useful.
\end{abstract}

Keywords:mindblindness, autism, mind reading architecture, impairment, Eye language interpreter, man machine interaction.

\section{INTRODUCTION}

A theory of mind is a representational set of abilities that allows one to mind read. It is the ability to attribute mental states to others, and use that to understand the actions and expressions of others within an intentional or goal-directed framework (Dennett in [11] refers to that as the intentional stance).

Facial movements and eye language in particular play a crucial role in recognizing basic emotions (such as happiness, sadness, disgust and fear) as well as "cognitive" or complex mental states (such as distrust, recognize, scheme, admire, interest, thoughtfulness, etc.) [9]. Using a digital video camera, the mind-reading computer ppt system analyzes a person's facial expressions in real time and infers that person's underlying mental state, such as whether he or she is agreeing or disagreeing, interested or bored, thinking or confused.

Prior knowledge of how particular mental states are expressed in the face is combined with analysis of facial expressions and head gestures occurring in real time. The model represents these at different granularities, starting with face and head movements and building those in time and in space to form a clearer model of what mental state is being represented. Software from Nevenvision identifies 24 feature points on the face and tracks them in real time. Movement, shape and colour are then analyzed to identify gestures like a smile or eyebrows being raised. Combinations of these occurring over time indicate mental states. For example, a combination of a head nod, with a smile and eyebrows raised might mean interest. The relationship between observable head and facial displays and the corresponding hidden mental states over time is modeled using Dynamic Bayesian Networks.

Current projects in Cambridge are considering further inputs such as body posture and gestures to improve the inference. We can then use the same models to control the animation of cartoon avatars. We are also looking at the use of mind-reading to support on-line shopping and learning systems.

The mind-reading computer system may also be used to monitor and suggest improvements in humanhuman interaction.

\section{HOW MIND READING IS DONE?}

\subsection{Futuristic head:}

The mind reading actually involves measuring the volume and oxygen level of the blood around the subject's brain, using technology called functional near-infrared spectroscopy

(fNIRS).

The user wears a sort of futuristic headband ${ }^{1}$ (see fig 1) that sends light in that spectrum into the tissues of the head where it is absorbed by active, blood-filled tissues. The headband then measures how much light was not absorbed, letting the computer gauge the metabolic demands that the brain is making.

\footnotetext{
${ }^{1}$ Headband is a type of headgear which a user can wear, fig 1 shows a user wearing head gear.
} 


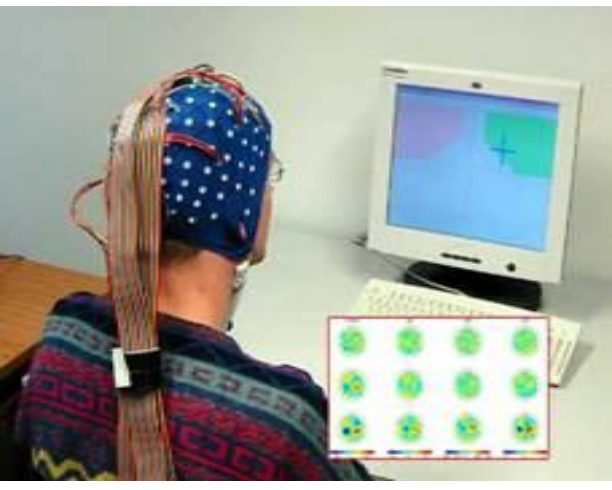

Figure 1

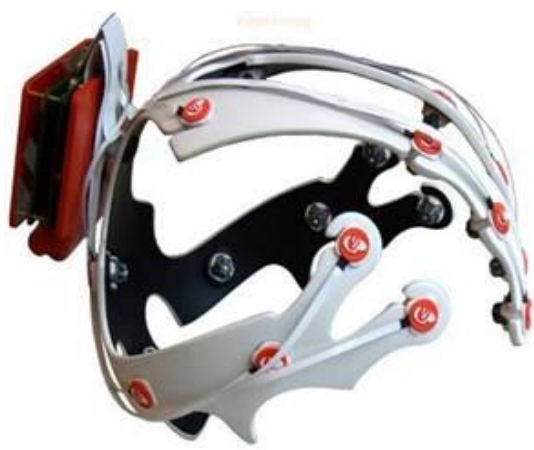

Figure 2

Future Headband

\subsection{Brain Chip:}

It is an independent processor linked to the neurocomputer built to house an artificial intelligence. The artificial intelligence program has access to the sensory data and information in the neurocomputer, and can "read" surface thoughts of the owner. Having a (or several) as advisor/secretary/partner is becoming more and more common, although most people realy on an external artificial intelligence system and a wireless neural connection[5]. It is not uncommon for users to get a motoric shunt to give the artificial intelligence the ability to control the body. Chips with monitoring artificial intelligence are sometimes used for or behavior correction in Landfall.

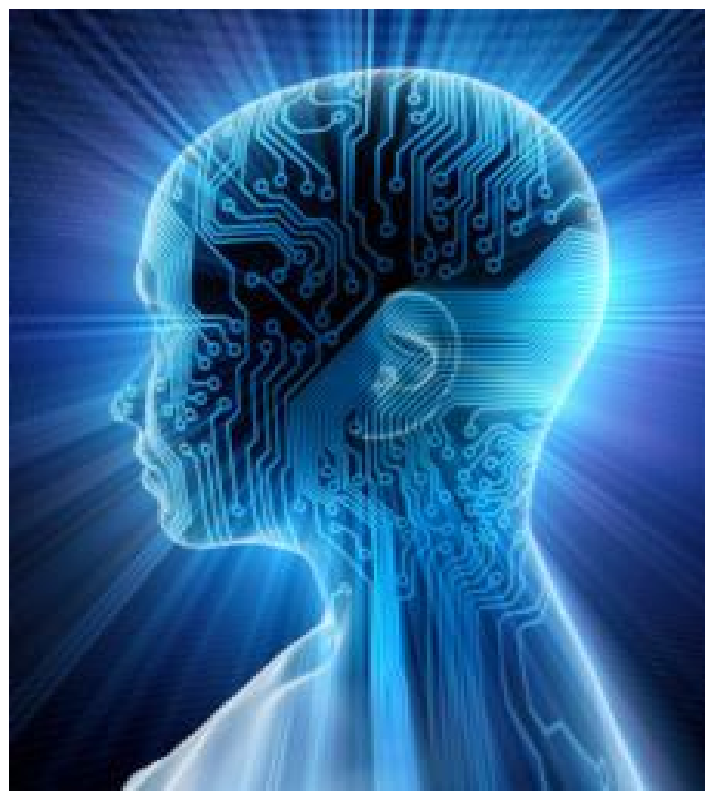

Figure 3

Scientists successfully implants chips that control Brain allowing thoughts, memory and behavior to be transferred from one brain to another brain. In a scene right out of a George Orwell novel, a team of scientists working in the fields of "neural engineering" and "Biomimetic MicroElectronic Systems" have successfully created a chip that controls the brain and can be used as a storage device for long-term memories. In studies the scientists have been able to record, download and transfer memories into other hosts with the same chip implanted. The advancement in technology brings the world one step closer to a global police state and the reality of absolute mind control.

\section{MINDREADING ARCHITECTURE}

While a number of researchers are building a theory of mind into humanoid robots [13] and [10] as a tool to test and evaluate developmental theories, we propose integrating a comparable model into mainstream interfaces as a novel approach to building social and emotion intelligence in interfaces. Seamless, non-obtrusive and implicit mind reading capabilities in mainstream interfaces could open new possibilities for man-machine interaction. In this section we introduce an architecture for developing Mindreading Interfaces. It integrates an automated eye language interpreter and a theory of mind model to enable the recognition and prediction of the user's mental state.

\section{Eye Language Interpreter:}

We pursue Baron-Cohen's research on a Language of the Eyes [9] to develop an automated eye language interpreter 
that recognizes eye expressions off image sequences and interprets them as mental states. We are interested in being

able to recognize a limited set of complex mental states that go beyond the 6 basic emotions. We use the Facial Action Coding System (FACS) developed by Ekman \& Friesen [12] to identify expressions of the eye. FACS is based on the enumeration of all action units of a face that causes facial movements. We identify head position and orientation, eye (and eye-brow) position and gaze actions units as the most relevant ones in addressing the problem of understanding eye expressions. We then develop a mapping between mental states and eye expressions.

\section{The Mindreading System}

We use Baron-Cohen's Mindreading System [6], which consists of four modular components for our implementation. We perform a number of projections specific to $\mathrm{HCI}$ to accommodate the differences in interaction format and modalities.

The Intentionality Detector (ID) interprets the selfpropelled motion of stimuli in terms of primitive volitional mental states of goal or desire. It builds dyadic representations, which specify the relation (desire or goal) between an agent and an object (or another agent). We suggest using stimuli from the pointing device to build the dyadic representations of ID.

Eye-Direction Detector (EDD) detects the presence of eyelike stimuli in the visual field, computes whether the eyes are looking at it or at something else, and interprets gaze direction as a perceptual state. EDD codes dyadic representational states of the form [Agent-looking at-me] and [Agent-looking at-not me]. The self is mapped to elements of the user's computing environment.

The Shared Attention Mechanism (SAM) is held to be necessary for the development and production of jointattention behaviors. SAM links ID to EDD by importing volitional terms from dyadic representations, into the relation slot of triadic representations, such that a person's goal or desire can be read from their eye-direction. SAM is also responsible for triggering ToMM.

The theory of Mind Mechanism or (ToMM) is responsible for our everyday ability to make sense of behavior in terms of mental states, and predicts an Agent's behavior on the basis of such states. It represents the full range of mental state concepts, and integrates mental state knowledge into a coherent and usable "theory" for humans to employ.

\section{Enabling Technologies: Automated} Facial FeatureAnalysis In order to be able to fully implement the Eye Language Interpreter and the Mindreading System, we need the services of a number of enabling technologies. We identify automated facial feature analysis as one of those technologies, and describe ongoing work on the implementation of specific work in that area. We are developing an automated facial feature tracking methodology that tracks spontaneous expression over time. The module should detect a face, locate features within the face, and then track the motion of those features across frames of an image sequence. The methodology is used to implement the Eye Language Interpreter and the Eye Direction Detector.

\section{RECOMMENDER METHOD}

Mind reading allows us to make sense of other people's behavior, predict what they might do next, and how they might feel. A lack of or impairment in mind reading abilities are thought to be the primary inhibitor of emotion and social understanding in people diagnosed with autism (e.g. Baron-Cohen et. al [6]). People employ a variety of nonverbal communication cues to infer underlying mental states, including voice, posture and the face. The human face in particular provides one of the most powerful, versatile and natural means of communicating a wide array of mental states. One subset comprises cognitive mental states such as thinking, deciding and confused, which involve both an affective and intellectual component [9].

Mindreading can be used for shopping purpose using mental states such as thinking, deciding, voice, posture and face. Suppose when we go in the new mall, we don't know anything about it like where is the Madam showroom or where is the Dunking Donuts parlor. So due to this reason we have to roam all over the mall to find a particular place. This over all headache can be overpower using mindreading technique. This can be understand by taking an example. When we go in a mall or in a shop there is scanner before entering in the mall. We can use the concept of mindreading in this scanner. We can use headband during this scanning process.

Mind reading involves measuring the volume and oxygen level of the blood around the subject's brain.So during scanning process, the user wears a sort of futuristic headband when he/shewalk through the scanner where thatsends light in that spectrum into the tissues of the head where it is absorbed by active, blood-filled tissues. The headband then measures how much light was not absorbed, letting the computer gauge the metabolic demands that the brain is making. When computer know the demands that the brain is making then by analyzing this he make a list of the showrooms or where ever user want to go with the floor numbers and with proper directions. So in this way our time save. No more roaming on different floors of mall to find a showroom or anything else.

\section{WHY MINDREADING?}

Imagine a future where we are surrounded with mobile phones, cars and online services that can read our minds and react to our moods. How would that change our use of technology and our lives?

Mind-reading can also support on-line shopping and learning systems. There are many uses of minreading(See table 1) 
Table 1 . Different working areas of mindreading

\begin{tabular}{|c|c|}
\hline $\begin{array}{l}\text { WORKING } \\
\text { AREAS }\end{array}$ & USES \\
\hline $\begin{array}{l}\text { MILITARY } \\
\text { AREAS }\end{array}$ & $\begin{array}{l}\text { A science fiction fantasy }- \text { the } \\
\text { "Thought Police" - where the } \\
\text { government reads people's memories } \\
\text { and thoughts and then rehabilitate } \\
\text { them through torture before they ever } \\
\text { even commit a crime based on a } \\
\text { statistical computer. }\end{array}$ \\
\hline $\begin{array}{l}\text { MEDICAL } \\
\text { AREAS }\end{array}$ & $\begin{array}{l}\text { 1. Mind-Reading Computer } \\
\text { Gives Voice, Movement to } \\
\text { the Paralyzed: New } \\
\text { technology may help those } \\
\text { who are locked inside their } \\
\text { own bodies (like in locked- } \\
\text { in-syndrome). } \\
\text { 2. Mind reader computer can } \\
\text { communicate with the } \\
\text { patients in coma: Canadian } \\
\text { researchers have developed } \\
\text { an interesting Computer } \\
\text { which can read mind and } \\
\text { could be helpful to } \\
\text { communicate with the } \\
\text { people who are in coma. } \\
\text { This can be called a } \\
\text { Computer Mind reader. }\end{array}$ \\
\hline GADGETS & $\begin{array}{l}\text { Mindreading technique can also used } \\
\text { in mobile phones, cars, keyboards and } \\
\text { mouse etc. }\end{array}$ \\
\hline
\end{tabular}

\subsection{Mind-Reading Computer Gives Voice, Movement to the Paralyzed:}

New technology may help those who are locked inside their own bodies. In locked-in syndrome, a condition in which people with normal cognitive brain activity suffer severe paralysis, often from injuries or an illness such as Lou Gehrig's disease. Boston University neuroscientist Frank Guenther works with the National Science Foundation's Center of Excellence for Learning in Education, Science and Technology (CELEST). Its purpose is to synthesize the experimental modeling and technological approaches to research in order to understand how the brain learns as a whole system. In particular, Guenther's research is looking at how brain regions interact, with the hope of melding mind and machine, and ultimately making life much better for people with "lockedin syndrome".

\subsection{Mind-reading program translates brain activity into words:}

Algorithms translated the brain activity associated with hearing 'Waldo', 'structure', 'doubt' and 'property' into recognizable words. Scientists have picked up fragments of people's thoughts by decoding the brain activity caused by words that they hear.

The remarkable feat has given researchers fresh insight into how the brain processes language, and raises the tantalizing prospect of devices that can return speech to the speechless. Though in its infancy, the work paves the way for brain implants that could monitor a person's thoughts and speak words and sentences as they imagine them. Such devices could transform the lives of thousands of people who lose the ability to speak as a result of a stroke or other medical conditions.

Experiments on 15 patients in the US showed that a computer could decipher their brain activity and play back words they heard, though at times the words were difficult to recognize.

\subsection{Mind reader computer can communicate with the patients in coma:}

Canadian researchers have developed an interesting Computer which can read mind and could be helpful to communicate with the people who are in coma. This can be called a Computer Mind reader[14]. A team of researchers from the University of Western Ontario were using the neuroimaging technique to read human thoughts brain activity while giving the specific answerin Yes or No.

In their study they asked very simple questions to the participants and told to concentrate only on the response either Yes or No. They were asked questions like "Are you married?" , "Do you like ice cream?" and answers the question which were asked in scanner by only concentrating upon the word they want to speak. By analyzing the activity of their brain they were able to decode the answers perfectly for every single person and for this time window is attached for communication with the brain computer interfaces answers in between $3 \mathrm{~min}$ of scanning. This technique is used to talk with the patients who are not able to response because of unconscious state of their mind.

\subsection{Mind reading computer can be used for police and military purpose:}

A science fiction fantasy - the "Thought Police" - where the government reads people's memories and thoughts and then rehabilitate them through torture before they ever even commit a crime based on a statistical computer analysis showing people with certain types of thoughts are likely to commit a certain type of crime in the future. We already pre-emptively invade nations and torture alleged terrorist suspects with absolutely no due process of law, so the idea 
of pre-emptively torturing a terrorist suspect before hand to prevent them from committing an act of terrorism in the future really isn't that far fetched of an idea.

\section{CONCLUSION}

Mindreading is the ability to infer other people's mental state and use that to make sense of and predict their behavior. A lack of or impairment in the theory of mind (mindblindness) is the primary inhibitor of emotion understanding and social intelligence in individuals with autism [7].In this paper we conclude how mind reading is done using some techniques. And different working areas of mindreading computer. A mind-reading architecture based on an Eye Language Interpreter and Baron-Cohen's Mindreading System is developed for the recognition and prediction of complex emotion states. And also proposed one more use of mind reading computer in real world. We believe that seamless, non- obtrusive and implicit mindreading capabilities in mainstream interfaces will open new possibilities for intelligent and effective interfaces.

\section{REFRENCES}

1. Mindreading computers-Wikipedia-the free encyclopedia.

2. Kevin brown , 2011. IBM research.

3. Ian Sample,2012. Mindreading translate brain activity into words.

4. Baron-Cohen, S. 1993. From attention-goal psychology to belief-desire psychology: the development of a theory of mind and its dysfunction. Understanding other minds: perspectives from autism. Baron-Cohen, S., Tager-Flusberg, H., and Cohen, D.J. Eds. Oxford University Press.

5. Mindtech Sweden, Mind control mind computer.

6. Baron-Cohen, S. 1994. How to build a baby that can read minds: Cognitive mechanisms in mindreading. Current Psychology of Cognition 13 (5): 513-552.

7. Baron-Cohen, S. 1995. Mindblindness: an essay on autism and theory of mind. MIT Press.

8. Julia Layton,2011.Have scientists found a way to read your mind?

9. Baron-Cohen, S., Wheelwright, S., and Jolliffe, T. 1997. Is There a "Language of the Eyes"? Evidence from Normal Adults, and Adults with Autism or Asperger Syndrome. Visual Cognition 4 (3): 311 331.

10. Deak, G., Fasel, I., and Movellan, J.R. 2001. The Emergence of Shared Attention: Using Robots to Test Developmental Theories. First International Workshop on Epigenetic Robotics: Modeling Cognitive Development in Robotic Systems.
11. Dennett, D. 1987. The Intentional Stance. MIT Press.

12. Ekman, P. and Friesen, W. 1978. Facial Action Coding System: A technique for the measurement of facial movement. Consulting Psychologists Press.

13. Scassellati, B. 2000. Models of Social Development Using a Humanoid Robot. Biorobotics. Webb, B. and Consi, T. Ed. MIT Press.

14. Mark prigg .Mind reading computer could communicate with the people in coma.

15. Paul bello,2012. Cognitive Foundations for a Computational Theory of Mindreading. 